\title{
REVIEW
}

\section{Monitoring healing of pressure ulcers: a review of assessment instruments for use in the spinal cord unit}

\author{
MS van Lis ${ }^{1}$, FWA van Asbeck ${ }^{2}$ and MWM Post ${ }^{2}$ \\ ${ }^{1}$ Bovenij Ziekenhuis, Department of Rehabilitation, Amsterdam and Rehabilitation Centre, Amsterdam, The Netherlands \\ and ${ }^{2}$ Rehabilitation Centre 'De Hoogstraat' and Rudolf Magnus Institute for Neuroscience, University Medical Centre, \\ Utrecht, The Netherlands
}

\begin{abstract}
Study design: Review.
Objectives: To identify the pressure ulcer healing assessment instrument that is the best choice for use in spinal cord injury rehabilitation.

Methods: Articles were retrieved from PubMed. Inclusion criteria were written in English, published up to December 2008 and describing instruments evaluated in more than one study. Search terms were pressure ulcer, wound healing, severity of illness index, reproducibility of results, sensitivity and specificity. Articles describing pressure ulcer staging scales and articles not describing clinimetric properties of an instrument were excluded. Validity, reliability, responsiveness and feasibility for routine clinical use were described of all instruments evaluated in two or more studies.

Results: Eleven instruments were described. Clinimetric information was incomplete for all instruments. Clinimetric information was most complete and promising for two instruments: 'ruler length and width' and the 'Sessing' scale. The ruler method showed good intra-rater and inter-rater reliability and good concurrent validity. The 'Sessing' scale has a moderate concurrent validity but was not tested for its responsiveness.

Conclusion: Further study of the clinimetric properties of pressure ulcer assessment instruments is necessary before the best instrument can be selected.

Spinal Cord (2010) 48, 92-99; doi:10.1038/sc.2009.146; published online 1 December 2009
\end{abstract}

Keywords: pressure ulcer; treatment outcome; review

\section{Introduction}

Pressure ulcers are a frequent secondary condition for individuals with spinal cord injury (SCI), with a long-term prevalence ranging from 15.2 to $30 \%{ }^{1,2}$ If pressure ulcers are present, these are often severe and take a long time to heal. Ulcers heal by wound contracture and replacement of normal tissue layers by granulation tissue, collagen and scar formation. ${ }^{3}$ To detect healing stagnation or deterioration, the 'Clinical Practice Guidelines' (CPG) recommend to evaluate the healing progress at least weekly using quantitative measures. ${ }^{4}$ Routine clinical assessment should include at least a measurement of length, width and depth, besides anatomical location, stage, exudat/odour, necrosis, undermining, sinus tracts infection, healing and wound margins. It is also recommended to modify the conservative treatment or to plan an operation if the ulcer shows no evidence of healing within $2-4$ weeks. $^{4}$

Correspondence: Dr FWA van Asbeck, Spinal cord unit, Rehabilitation Centre De Hoogstraat, Rembrandtkade 10, Utrecht NL-3583 TM, The Netherlands. E-mail: f.v.asbeck@dehoogstraat.nl

Received 8 July 2009; revised 16 September 2009; accepted 20 September 2009; published online 1 December 2009
Up to the present, there is no worldwide consensus about the best instrument to measure pressure ulcer healing in SCI rehabilitation. It is, however, already clear that some instruments are not suitable. Staging or grading scales as the National Pressure Ulcer Advisory Panel staging system, European Pressure Ulcer Advisory Panel scale and Stirling scale are not suitable because these classification systems were designed to rate the severity of a pressure ulcer and not its healing. ${ }^{5,6}$ In 1997, it was concluded that no ideal instrument to measure pressure ulcer healing was available. ${ }^{3,7}$ These and later reviews, however, described only part of available instruments or described instruments used for other kinds of wounds. ${ }^{3,7-10}$

To guide a choice from the large number of instruments described in the literature, we performed a literature review of pressure ulcer assessment instruments to describe their validity, reliability and responsiveness. We also investigated which instrument will be most feasible with respect to time (preferably $<1$ min for measurement and registration) and costs for use in routine bedside practice in a medical unit specialized in inpatient and outpatient SCI rehabilitation. 


\section{Methods}

The primary data source was PubMed. Articles were selected using the MeSH Major Topic 'pressure ulcer', covering the following terms: pressure ulcers, bedsore(s), bed sore(s), decubitus ulcer(s) and pressure sore(s). The searches consisted of this MeSH Major Topic and one of the following four other MeSH terms: wound healing, severity of illness index, reproducibility of results, and sensitivity and specificity. Articles were included that were written in English and published up to 31 December 2008. Excluded were review articles, articles on staging or grading scales, articles without description of any clinimetric properties of the used pressure ulcer assessment instrument, articles in which the instrument was evaluated using plaster wound models and articles that described a mixed population, for example, patients with pressure ulcers and patients with leg ulcers.

References of included articles were checked, and additional articles were included if clinimetric properties of pressure ulcer assessment instruments were described. Finally, for each of the included instruments, we performed a Pubmed search for additional articles about that instrument, and these articles were also included in this review if they described clinimetric properties. As these searches revealed many different instruments, we selected only instruments that were evaluated in more than one publication.

To evaluate concurrent or criterion validity, we considered correlation coefficients (Pearson, Spearman) $>0.60$ as support $(+), 0.30-0.60$ as partial support $( \pm)$ and $<0.30$ as no support (-). For multidimensional scales, evidence for construct validity was present if the multidimensional structure was confirmed by factor or principal component analysis $(+)$. For one-dimensional scales, this criterion was not applicable (na). Inter-rater and intra-rater reliability (Kappa, Pearson, ICC) was good if $>0.80(+)$, partial if $0.70-$ $0.80( \pm)$ or insufficient if $<0.70(-)$. Some studies evaluated reliability using the coefficient of variation. There are no norms for interpretation of this statistic available, making it difficult to interpret. In this review, a variation of $6 \%$ or less was rewarded with a plus. Evidence for responsiveness was found if statistically significant improvements were reported on follow-up measurements and were related to the time after baseline measurement (number of weeks). Feasibility was described as time needed to rate one ulcer (minutes), the types of ulcers for which the test is applicable (types I-IV), the amount of training needed before application (minutes) and the costs of the test, software or hardware in US dollars (USD).

\section{Results}

The combination of 'pressure ulcer' with the MeSH term wound healing revealed 858 articles, with the term severity of illness index 289, with the term reproducibility of results 190 and with the term sensitivity and specificity 155 articles. Some articles appeared more than once in the search results because they contained two or more of these MeSH terms.

After we applied the exclusion criteria, 33 articles remained. We included seven additional articles from the literature list of these 33 articles. Two of these seven articles were published in a journal, which is not indexed in PubMed. The five other articles were available in PubMed but did not include the MeSH terms we used. To make sure that we had not missed other relevant articles, we performed a final series of searches using the MESH term 'pressure ulcer' in combination with MESH terms from these last articles: pressure ulcer/radiography, pressure ulcer/economics, weights and measures, nursing assessment and monitoring, physiologic. We found no further relevant articles using these terms.

The 40 included articles described 21 instruments (Table 1). Only 11 of these instruments were described in more than one publication. A summary of their clinimetric properties is provided in Table 2 and extensive information on each instrument is provided in Table 3.

Volume was assessed with saline, alginate or with a ruler measuring length, width and depth. When volume was assessed with saline, a sheet was applied tightly over the wound and filled with physiological saline $(\mathrm{NaCl})$ gel $^{11}$ or fluid $^{12}$ through a hole in the centre of the sheet. The volume needed was registered.

When volume was assessed with alginate, the compound was applied in the (rinsed) wound while still fluid. The moulds were extracted and weighted, and volume was computed by dividing weight by density. ${ }^{13,14}$ Otherwise moulds were immersed in water and volumetric displacement was measured. This was compared with measurement of the volume of the moulds by nuclear magnetic resonance spectrometer. $^{15}$

For assessment of the volume by measuring length, width and depth, a horizontal cross-arm ruler with a vertical arm for crater wounds was developed. ${ }^{16}$ The surface area or volume of the wound was calculated with different formulas.

All three-volume assessment instruments showed concurrent validity (Table 2). Only the 'filling material saline' was

Table 1 Overview pressure ulcer assessment instruments

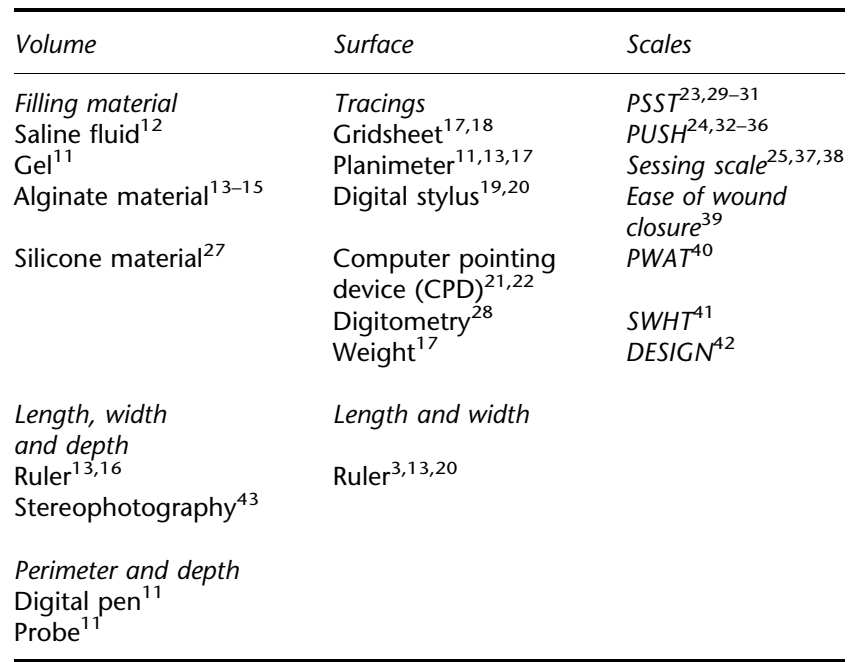

Abbreviations: PSST, Pressure Sore Status Tool; PUSH, Pressure Ulcer Scale for Healing. 
Table 2 Clinimetric aspects of pressure ulcer assessment instruments

\begin{tabular}{|c|c|c|c|c|c|c|c|c|c|}
\hline \multirow[t]{2}{*}{ Instrument } & \multicolumn{2}{|c|}{ Validation } & \multicolumn{2}{|c|}{ Reliability } & \multicolumn{4}{|c|}{ Practicality } & \multirow{2}{*}{$\begin{array}{l}\text { Response } \\
\text { Response }\end{array}$} \\
\hline & Construct ${ }^{\mathrm{a}}$ & Concurrent $^{\mathrm{b}}$ & Inter-rater & Intra-rater ${ }^{\mathrm{c}}$ & Type $e^{\mathrm{d}}$ & Time $^{\mathrm{e}}$ & $\operatorname{Cost}^{\mathrm{f}}$ & Training $^{\mathrm{g}}$ & \\
\hline \multicolumn{10}{|l|}{ Volume } \\
\hline Filling material saline & NA & + & - & + & III-IV & $<15$ & $?$ & $?$ & $?$ \\
\hline Filling material alginate & NA & + & $?$ & $?$ & III-IV & $?$ & $?$ & $?$ & 4 \\
\hline Ruler length, width and depth & NA & + & $?$ & $?$ & I-IV & $?$ & $?$ & $15-30$ & 3 \\
\hline \multicolumn{10}{|l|}{ Surface } \\
\hline Tracings with grid sheet & NA & $?$ & + & + & I-II & $5-7$ & 2 & $?$ & $?$ \\
\hline Tracings with planimetry & NA & + & + & + & I-II & $?$ & $?^{i}$ & $?$ & 2 \\
\hline Tracings with digital stylus & NA & + & $?$ & + & I-II & $?$ & $0.20^{\mathrm{j}}$ & $?$ & $?$ \\
\hline Tracings with CPD & NA & $?$ & $?$ & + & I-II & $?$ & $?$ & $?$ & $?$ \\
\hline Ruler length and width & NA & + & + & + & I-II & $?$ & 2 & $?$ & 2 \\
\hline \multicolumn{10}{|l|}{ Scales } \\
\hline PSST & + & $?$ & \pm & + & I-IV & $10-15$ & $?$ & 30 & $?$ \\
\hline PUSH & \pm & + & $?$ & $?$ & I-IV & $1-5$ & $?$ & 50 & $2 / 4$ \\
\hline Sessing scale & NA & $\pm /+$ & + & + & I-IV & 1 & $?$ & 30 & $?$ \\
\hline
\end{tabular}

Abbreviations: AHCPR, Agency for Health Care Policy and Research; NPUAP, National Pressure Ulcer Advisory Panel; PSST, Pressure Sore Status Tool; PUSH, Pressure Ulcer Scale for Healing.

${ }^{a}$ Construct validity: +, multidimensional structure confirmed by factor or principal component analysis; -, structure not confirmed by factor or principal component analysis; NA, not applicable (one-dimensional scale).

${ }^{\mathrm{b}}$ Concurrent validity: correlation coefficients (Pearson, Spearman):,$+>0.60$ strong correlation;, $\pm 0.30-0.60$ partial correlation.,$-<0.30$ no correlation.

${ }^{\mathrm{C}}$ Kappa, Pearson, ICC:,$+>0.80$ good;, $\pm 0.70-0.80$ partial;,$-<0.70$ insufficient; ?, unknown/unclear.

dType of ulcer: NPUAP/AHCPR stages I-IV.

${ }^{\mathrm{e}}$ Time per pressure ulcer in minutes.

${ }^{f}$ Cost in USD per test and instrument.

${ }^{9}$ Training in minutes.

${ }^{\mathrm{h}}$ Responsiveness: in weeks after baseline statistical difference detected.

iPlanimeters can be very expensive. ${ }^{17}$

jPhotographic method: camera and film processing > 850-1000 USD additional.

tested for reliability. The reliability with dental-moulding material was insufficient, especially for wounds with deep sinus tracts or deep undermining or shallow wounds. ${ }^{3}$ The 'ruler length, width and depth' method is the most practical instrument of the three. Responsiveness was evaluated for 'filling material saline' and 'ruler length, width and depth' and was better for the last one.

Surface was assessed with a gridsheet, planimetry, digital stylus, computer pointed device or ruler.

The gridsheet was used in two different ways. A sterilized transparency film was placed over the wound and the wound's perimeter was traced on a metric graph paper and the number of $\mathrm{mm}^{2}$ within the tracing was counted. ${ }^{17}$ Otherwise the area of the wound surface was outlined from a photograph on a transparent wound diagram consisting of a $\mathrm{mm}^{2}$-scaled grid. The enclosed area was calculated by observers. $^{18}$

Planimetry was also used in two different ways. A planimeter was used to determine ulcer surface from wound tracings on a grid sheet ${ }^{11,13,17}$ or tracing of the ulcer was drawn on the photograph before using the planimeter.

Digital stylus was used on a transparency on which the ulcer margins were traced with an inedible pen or on a photograph of the wound. The tracing was outlined using the stylus of a tablet digitizer interface with a computer. The area was calculated using different software programs. ${ }^{19,20}$

Computer pointed device was used after a record of the wound taken by video with reference scales at right angles or a digital camera. From the computer image the surface area is traced with a tracker ball, and the surface area is calculated by the number of pixels ${ }^{21}$ or a software program. ${ }^{22}$

Rulers are used in different forms calculating the area with different formulas. ${ }^{3,13,20}$ Measurements were taken according to the CPG. ${ }^{4}$

From the five surface assessment instruments described in Table 2, only the 'wound tracings with planimetry' and the 'ruler length and width' showed concurrent validity and were also tested for their inter-rater and intra-rater reliability. Reliability of the 'wound tracings with planimetry' was evaluated with the coefficient of variation. The 'wound tracings with planimetry' method has practical problems: tracing the wound area is often difficult as the ulcer margins are not always clearly visible. Besides that a planimeter can be prohibitively expensive. The 'ruler length and width' method, using Kundin device, is cheap, but it slightly over-estimated the areas obtained by planimetry (mean difference of about $1.5 \mathrm{~cm}^{2}$ in ulcers of $1.2-$ $\left.61.6 \mathrm{~cm}^{2}\right) .^{13}$ The responsiveness to change was evaluated in both instruments. Wound tracings with a planimeter and ruler length and width were both sensitive to measure wound change early in treatment as they revealed significant percentage differences 2 weeks after measuring the baseline size of wounds $<10 \mathrm{~cm}^{2}$ and 4 weeks after measuring the baseline size of wounds $\geqslant 10 \mathrm{~cm}^{2}$. ${ }^{13}$ A very high correlation between these two methods was found $(r=0.979$, $P<0.001) .^{13}$ 
Table 3 Detailed description of 11 pressure ulcer assessment instruments: (a) volume; (b) surface; (c) scales

(a)

Filling material: saline

First publication ${ }^{\mathrm{a}}$

Method $(\mathrm{NaCl})$

1: Validation

2: Reliability

3: Practicality

4: Responsiveness Not evaluated

Berg et $a l^{12}$

Sheet applied tightly over the wound and filled with physiological saline gel ${ }^{11}$ or fluid ${ }^{12}$ through a hole in the centre of the sheet. Volume needed was registered

Concurrent: Gel 40\%/ $\mathrm{NaCl} 60 \%, r=0.84, P<0.01$ (wound tracings with planimeter), $r=0.76, P<0.01$ (perimeter with digital pen), $r=0.69, P<0.01$ (depth with probe) ${ }^{11}$

Inter-rater: reproducibility coefficient of variation (CoV) is $19 \%^{11}$

Intra-rater: repeatability coefficient of variation (CoV) is $6 \% .^{11}$

'Satisfactory' reproducibility without providing figures ${ }^{12}$

Cost: not described

Time test: $<15 \mathrm{~min}^{11}$

Type: test not applicable for NPUAP/AHCPR stages I and II ulcers. With gel no leakage to interfere with measurement ${ }^{11}$

Training: not described

Filling material: alginate

First publication $^{\mathrm{a}} \quad$ Resch et al. $^{14}$

Method

Compound is applied in (rinsed) wound while still fluid. Molds extracted and weighted, dividing weight by the density. ${ }^{13,14}$ Or molds were immersed in water and volumetric displacement was measured and passed through nuclear magnetic resonance spectrometer, which measured volume ${ }^{15}$

1: Validation

2: Reliability

3: Practicality

Concurrent: $r=0.892$ (length, width and depth with ruler). ${ }^{13}$ Water displacement, $r=0.96$ (computed volume)

Inter-rater/intra-rater: not evaluated. Note: 'small variations since levelling of surface is done by eye'

Cost: not described

Time test: 'quick'14

Type: test not applicable for NPUAP/AHCPR stages I and II ulcers

Training: 'easy to learn' ${ }^{14}$

4: Responsiveness Statistical significant change in wound size at week $4(P<0.05)$ for wounds $\geqslant 10 \mathrm{~cm}^{2}$. No statistical difference in weeks $1-4$ in wounds $<10 \mathrm{~cm}^{2} 13$

Length, width and depth: ruler

First publication $^{\mathrm{a}} \quad$ Kundin $^{16}$

Method

Different: (1) longest dimension (a) and longest dimension (b) perpendicular to the first dimension and depth measured at the deepest point $(c)$. Volume $=(2 / 3) \prod(a / 2)(b / 2) c .^{13}(2)$ Horizontal cross-arm ruler with a vertical arm for crater wounds: area $=$ length $\times$ breadth $\times 0.785$ and volume $=$ area $\times$ depth $\times 0.327$ (Kundin device) ${ }^{16}$

1: Validation

2: Reliability

3: Practicality

Concurrent: $r=0.892$ (filling material alginate). ${ }^{13}$ 'Correlates well in trials ${ }^{16}$

Inter-rater: not evaluated Intra-rater: not evaluated

Cost: not described

Time test: not described

Type: applicable for NPUAP/AHCPR stages I-IV ulcers

Training: 15-30 min of instruction and return demonstration needed for accurate results ${ }^{16}$

4: Responsiveness

Significant wound closure detected at week 3 for wounds $\geqslant 10 \mathrm{~cm}^{2}$ and week 4 for wound $<$ and $\geqslant 10 \mathrm{~cm}^{2} 13$

(b)

Tracings: gridsheet

First publication

Method

Bohannon and Pfaller ${ }^{17}$

Different: (1) placing sterilized transparency film over the wound and tracing the wound's perimeter. Tracing the outline of the tracing on metric graph paper and counting the number of $\mathrm{mm}^{2}$ within the tracing. ${ }^{17}$

(2) From the photograph, the area of the wound surface was outlined on a transparent wound diagram consisting of a mm ${ }^{2}$ scaled grid. The enclosed area was calculated by observers ${ }^{18}$

1: Validation

2: Reliability

3: Practicality

Not described

Inter-rater: mean difference was $3.9 \%$ for 10 pairs of tracings. ${ }^{17}$ ICC $0.99^{18}$

Intra-rater: ICC $0.99^{18}$

Cost: metric graph paper of negligible cost, ${ }^{17} \$ 2$ for 1 measurement ${ }^{18}$

Time test: 5 min for each tracing, ${ }^{17}$ time required for photography and tracing of a pressure ulcer is $<7 \mathrm{~min}^{18}$

Type: photographs and tracings are two-dimensional and uniplanar, which may distort three-dimensional multiplanar wound surfaces $^{18}$

Training: 'simple to learn'18

4: Responsiveness

Not evaluated

Tracings: planimetry

First publication

Method

Bohannon and Pfaller ${ }^{17}$

Different: (1) a planimeter was used to determine ulcer surface areas from wound tracings ${ }^{11,13,17}$

(2) tracing of the ulcer was drawn on the photograph before using the planimeter ${ }^{13}$

1: Validation

Concurrent: wound tracings with planimeter $r=0.979, P<0.001$ (length and width), $r=0.915$ (length and width for wounds $<10 \mathrm{~cm}^{2}$ ), $r=0.964$ (length and width for wounds $\geqslant 10 \mathrm{~cm}^{2}$ ), $r=0.963$ (photographic tracings with planimeter), ${ }^{13} r=0.84$, $P<0.01$ (saline gel). ${ }^{11}$ Photographic tracings with planimeter $r=0.971, P<0.001$ (length and width), $r=0.963$ (wound

tracings with planimeter), $r=0.989 P<0.001$ (length an width photographs) ${ }^{13}$

2: Reliability
Inter-rater: mean difference was $3,6 \%$ for 10 pairs of tracings. ${ }^{17}$ Reproducibility coefficient of variation $=3 \%{ }^{11}$

Intra-rater: repeatability coefficient of variation $=2 \%{ }^{11}$ 
Table 3 Continued

\begin{tabular}{|c|c|}
\hline 3: Practicality & $\begin{array}{l}\text { Cost: planimeters can be very expensive }{ }^{17} \\
\text { Time test: not described } \\
\text { Type: determining the area was often difficult as the ulcer margins were not always clearly visible }{ }^{13} \\
\text { Training: not described }\end{array}$ \\
\hline 4: Responsiveness & $\begin{array}{l}\text { Wound tracings with planimeter: significant percentage differences at weeks } 2,3 \text { and } 4 \text { for wounds }<10 \mathrm{~cm}^{2} \text { and at week } 4 \text { for } \\
\text { wounds } \geqslant 10 \mathrm{~cm}^{2} 13\end{array}$ \\
\hline
\end{tabular}

Tracings: digital stylus First publication ${ }^{\text {a }}$ Method

Griffin et al. ${ }^{19}$

Different: (1) transparency was placed directly over the ulcer and margins were traced with an indelible pen (transparency method) or (2) wound was photographed using colour slide film. The slide was projected onto paper and focus was adjusted until the image of the metric ruler in the slide exactly matched the original ruler. Margins were then traced with a pen (photographic method). After method (1) or (2), each tracing was outlined using the stylus of a tablet digitizer interfaced with a computer. Area was calculated using software program (CADD 3.0). ${ }^{19}$ (3) As method (1), area was calculated using another software program (Zeiss Interactive Digital Analysis System). ${ }^{20}$ (4) Wound was photographed using slide film. Image was placed on digitizing tablet and wound margins were traced with a stylus. Area was calculated as method $(3)^{20}$

1: Validation Concurrent: single occasion: $r=0.993, P<0.0001,5$-day interval during 20-day: $r=0.996-0.999$ (transparency vs photographic) $^{19}$ $r=0.964, P<0.001(\text { method } 3 \text { vs } 4)^{20}$

$r=0.948, P<0.001$ (method 3 vs Kundin) $^{20}$

$r=0.927, P<0.001$ (method 4 vs Kundin) ${ }^{20}$

2: Reliability Inter-rater: not described

Intra-rater ICC $=0.999$ (mean of three measurements), ICC $=0.998$ (single measurement) (2; transparency method).

ICC $=0.999$ (mean of three measurements), ICC $=0.995$ (single measurement) (2; photographic method)

3: Practicality cost $\$ 36$ for a box of 25 transparencies ${ }^{19} \$ 0.20$ for a piece of acetate ${ }^{20}>\$ 850$ for camera, film and film processing ${ }^{19}>\$ 1000$ for camera, lens and battery pack ${ }^{20}$

Time test: more time was required in photographic method compared with transparency method. ${ }^{19}$ Method 4: the additional time needed to measure and process photo images detracts from its clinical usefulness ${ }^{20}$

Type: excluded: pressure sores extended into periosteum ${ }^{20}$

Training: methods 3 and 4 require some training ${ }^{20}$

4: Responsiveness

Not evaluated

Tracings: CPD

First publication ${ }^{\mathrm{a}}$ Method

1: Validation
2: Reliability

3: Practicality

4: Responsiveness

Palmer et al..$^{21}$

(1) Record the ulcer with a video camera with reference scale at right angles. From the computer image the surface area is traced with a tracker ball. Number of pixels in this area is determined ${ }^{21}$

(2) Ulcer with scale recorded by digital camera $(1524 \times 1012$ pixel). Manual outlining of ulcer area with computer mouse. Computerized analysis of area (software Foster and Findlay Associates) ${ }^{22}$

Concurrent: not evaluated

Inter-rater: not evaluated

Intra-rater: coefficient of variation ulcer area 4.76/5.53\% (within batch) 4.61/5.43/5.72\% (between batch). ${ }^{22}$ Coefficient of variation between 0.2 and $3.6 \%{ }^{21}$

Cost: not described

Time test: not described

Type: heels are a curved surface and reproducible images were impossible to achieve. ${ }^{22}$ Only two ulcers were tested ${ }^{21}$

Training: To minimize errors, the camera should be within a $10^{\circ}$ of the right angle to the ulcer ${ }^{21}$

Not evaluated

Length and width: ruler First publication ${ }^{\mathrm{a}}$ Method

Thomas and Wysocki ${ }^{20}$

Different: a cross-ruler: area $=$ length $\times$ breadth $\times 0.785$ (Kundin), ${ }^{20}$ product of the two largest diameters. ${ }^{3}$ Longest dimension (a) and longest perpendicular dimension (b) put in formula: area $=\prod(\mathrm{a} / 2)(\mathrm{b} / 2){ }_{1}^{13}$ photo with a calibration ruler ${ }^{13}$

1: Validation

Concurrent: Kundin device, $r=0.948, P<0.001$ (outline traced on acetate and digital analysis system), $r=0.927, P<0.001$ (photo and digital analysis system). ${ }^{20}$ Two largest diameters Pearson's $r=0.931, P<0.0000$ (tracings on plastic followed by planimetry). ${ }^{3}$ Two longest dimension in formula $r=0.979, P<0.001$ (wound tracings with planimeter), $r=0.915$ (wound tracings with planimeter for wounds $<10 \mathrm{~cm}^{2}$ ),$r=0.964$ (wound tracings with planimeter for wounds $\left.\geqslant 10 \mathrm{~cm}^{2}\right), r=0.971$, $P<0.001$ (photographic tracings with planimeter). ${ }^{13}$ Two longest dimensions in formula $r=0.979$ (planimetry measurements on transparent material) and $r=0.971, P<0.001$ (photographic tracings)

Inter-rater: Pearson's $r=0.9774, P<0.0000^{3}$

2: Reliability

3: Practicality

Intra-rater: for all wound measurements, there was no $>10 \%$ measuring error. ${ }^{20}$ Pearson's $r=0.9923, P<0.0000^{3}$

Cost: Kundin ruler $\$ 2^{20}$

Time test: not described

Type: Kundin consistently underestimated wound area, especially large and irregularly shaped wounds ${ }^{20}$

Training: require some training ${ }^{20}$

4: Responsiveness

Significant percentage differences at weeks 2,3 and 4 for wounds $<10 \mathrm{~cm}^{2}$ and at week 4 for wounds $\geqslant 10 \mathrm{~cm}^{2} 13$

(c)

Pressure Sore Status Tool (PSST)

First publication $^{\text {a }} \quad$ Bates-Jensen et al. ${ }^{23}$

Method Wound inspection with measurements (length $\times$ width, undermining). Rating with PSST sheet

Items

Score 13-65 (1-5 scale) 
Table 3 Continued
1: Validation $\quad$ Content: Established by a panel of 20 experts and refined by a nine-expert panel of judges. They rated relevance of 15 items on tissue, epithelialization a 4-point scale 23
Construct: factor analysis: all 13 items on the PSST correlated with the total PSST score. Four factors explained $64 \%$ of total variance in PSST score ${ }^{29}$ : refinement not done after factor analysis
Concurrent: with NPUAP stage or with only one item: PSST total score $r=0.606, P=0.001$ (NPUAP stage diagnosis). ${ }^{29} r=0.55$ PSST demonstrates the ability to discriminate between partial (I-II) and full-thickness (III-IV) NPUAP stage ${ }^{30}$
Subscale item depth with stage AHCPR $r=0.91^{31}$
Predictive: initial PSST score vs time-to-heal $(r=0.33, P=0.001)^{29}$
2: Reliability Inter-rater: mean reliability coefficient Pearson $r=0.915$ by enterostomal therapy nurses. ${ }^{23}$ Mean reliability for practitioners 0.78. ${ }^{31}$ Practitioners: two physical therapists, seven licensed practical nurses and six registered nurses, mean experience with wounds 8 years
Intra-rater: mean reliability coefficient Pearson $r=0.975$ by enterostomal therapy nurses. ${ }^{23}$ Mean reliability for practitioners $0.89^{31}$ Costs: use a ruler and transparent metric measuring guide with concentric circles divided into four (25\%) pie-shaped quadrants. Computer optional, cost unknown
Time test: without computer estimated $10-15 \mathrm{~min}^{8}$ with optional computer- program quicker ${ }^{30}$ : computer-based system for relating changes in wound status (PSST) to patient characteristics and treatment interventions
Type: applicable to all kind of pressure ulcers (comment author)
Training: 30 min training session, ${ }^{23}$ one-page sheet of instructions ${ }^{29}$ Two-hour computer training (WIS) ${ }^{30}$ estimated 70 min training 8
4: Responsiveness Not evaluated

15 items: 2 non-scored items: location and shape. 13 scored items: size, depth, edges, undermining, necrotic tissue type and amount, exudate type and amount, skin colour surrounding wound, peripheral tissue oedema and induration, granulation

Pressure Ulcer Scale for Healing (PUSH)

First publication $^{\text {a }} \quad$ Sussman $^{44}$

Method

Wound inspection with measurements (greatest length and width) using a ruler

Items

Originally consisting of three weighted items: surface area (five categories), exudate (four categories), appearance predominant tissue (four categories) with the sum score $8-34 .{ }^{33}$ To increase the sensitivity of the tool, the original authors 'the PUSH Task Force' adapted the instrument in 2001; one item was refined (appearance), another item expanded (surface area, 10 categories) and the items non-weighted. ${ }^{24}$ Sum score $0-14$

1: Validation

Content: review of the literature and expert opinion ${ }^{33}$

Construct: principal component (PC) analysis indicated that surface area, appearance and exudate amount of the ulcer define best model of healing $(P \leqslant 0.01)$

PC analysis: weighted items explained $55-65 \%$ of the variation in the data at each time point ${ }^{33}$

PUSH variables (unmodified) provided the best model of healing and account for $58-74 \%$ variation (10-week period) ${ }^{24}$

PC analysis with modified PUSH: variables account for $39-57 \%$ variation (12-week period) ${ }^{24}$

Concurrent: PSST (weeks 1-5): Pearson's $r=0.72-0.95(P=0.000)$. Acetate tracings with digitizing tablet (weeks $1-5)$ : Pearson's $r=0.70-0.83(P=0.000-0.001)^{35}$

Predictive: not reported data

2: Reliability

3: Practicality

'Agreement among the personnel was $>95 \%$ '. ${ }^{24}$ No data shown

Cost: unknown

Time test: $1 \mathrm{~min}^{24}$ to $5 \mathrm{~min}^{8}$ calculating scores weekly or biweekly for each patient is very time intensive, monthly data forms available $^{34}$

Type: wound healing is not adequately captured in small wounds with depth and large wounds $>24 \mathrm{~cm}^{236}$

$\begin{array}{ll}\text { 4: Responsiveness } & \text { Training: a review article }{ }^{8} \text { estimates that training for the original PUS } \\ & \text { Statistical difference: week } 0 \text { vs } 4 / 6 / 8^{\mathrm{b}} \text { and week } 2 \text { vs } 8^{\mathrm{b}}(P<0.05) \\ & \text { Statistical difference: week } 0 \text { vs } 2 / 4 / 6 / 8^{\mathrm{c}} \text { and week } 2 \text { vs } 8^{\mathrm{c}}(P<0.05)\end{array}$

Original PUSH: over a 6 -week period PUSH accounted for $39 \%$ of the variation $(P<0.001)$. Score was significantly different

$(P<0.05)$ between week 1 vs $2-10,2$ vs $3-10,3$ vs $5-10$ and 5 vs $7-10$. Weeks 6 through 10 were not statistically different ${ }^{24}$

Modified PUSH: over a 12-week period PUSH accounted for $31 \%$ of the variation $(P<0.001)$ of total healing. Score was significantly different $(P<0.05)$ between week 1 vs $2-12,2$ vs $3-12,3$ vs $5-12,4$ vs $5-12$ and 6 vs $10-12^{24}$

Total PUSH scores decreased significantly from week 1 through 5 among the healed ulcers $(\mathrm{df}=4 ; \mathrm{F}=5.901 ; P=0.001)$, but did not decrease significantly among the unhealed ulcers. The healed ulcers revealed significant differences in total PUSH scores between weeks $4-5(\mathrm{df}=1 ; \mathrm{F}=7.364 ; P=0.024)^{35}$

Comments

The opinion of the audience: some dressings make item 'exudate' difficult to categorize ${ }^{32}$

Sessing

First publication ${ }^{\mathrm{a}}$

Method

Items

1:Validation
Ferrell et al. ${ }^{37}$

Wound inspection

One 7-point scale with descriptions of the observed pressure ulcer

Content: a panel of five clinical nurse specialists evaluated the scale with regard to conceptual framework, content and hierarchy ${ }^{37}$ Construct: not evaluated

Concurrent: addressed with Shea and diameter. Initial Sessing score: $r=0.52 P<0.0001$ (initial Shea scale), $r=0.35 P<0.001$ (diameter) $^{37}$

Change in Sessing score: $r=0.90, P<0.0001$ (change Shea scale), $r=0.65 P<0.0001$ (change diameter) ${ }^{37}$

Predictive: in a multivariate regression analysis, the association between the initial Sessing score with healing was significant at the 0.05 level $^{38}$

Inter-rater: weighted kappa 0.80 (nurses with wound experience) ${ }^{37}$

Intra-rater: weighted kappa $0.90(N=10)$ and $0.84(N=50)$ (nurses with wound experience) ${ }^{37}$ 
Table 3 Continued

\begin{tabular}{ll}
\hline 3: Practicality & Cost: not described \\
& Time test: not described in the original article, ${ }^{37}$ estimated 1 min in review \\
& Type: applicable to all kind of pressure ulcers (comment author) \\
& Training: easy to learn for experts, ${ }^{37}$ estimated 30 min $^{8}$ \\
4: Responsiveness & Not evaluated
\end{tabular}

Abbreviations: AHCPR, Agency for Health Care Policy and Research; NPUAP, National Pressure Ulcer Advisory Panel.

${ }^{a}$ First publications describing clinimetric aspects in PubMed.

${ }^{\mathrm{b}}$ Original items weighted.

${ }^{\mathrm{c} O r i g i n a l}$ items non-weighted. ${ }^{33}$

The following three scales were included: the 'Pressure Sore Status Tool (PSST)', 'Pressure Ulcer Scale for Healing (PUSH)' and the 'Sessing scale'.

The PSST assesses 13 domains in 5 categories each. Size and undermining are measured before categorization, while the other domains are descriptive. Location and shape are added as non-scored items. ${ }^{23}$ The 'PSST' assesses the domains recommended by the CPG except for anatomical location and stage.

The PUSH assesses three domains. It categorizes the surface by multiplying greatest length and width and adds this to categories for exudate amount and tissue type. ${ }^{24}$ The PUSH assesses only the CPG-domains size, exudates/odour and healing.

The Sessing scale is a description of the wound in seven categories without measurements. ${ }^{25}$ It assesses the CPGdomains exudates/odour, necrosis, infection, healing and wound margins but gives no measurements.

All three scales showed clinimetric problems. The 'PSST' used staging scales to prove its concurrent validity. Further problems were the time needed for testing and lack of data on responsiveness.

The 'PUSH' scale has not been evaluated for reliability and is not practical to use in small wounds with depth and large wounds. However, it is the only scale tested for its responsiveness.

The 'Sessing scale' has a moderate concurrent validity and was not tested for responsiveness. It is easy to learn and quick to use.

\section{Discussion}

At least 21 different pressure ulcer assessment instruments have been published to date. They can be divided into volume instruments, surface instruments and scales (Table 1). Their clinimetric properties, however, are poorly described (Table 2). From the PubMed literature until 2009, we can conclude that no pressure ulcer assessment instrument has been completely tested for validity, reliability and responsiveness. In addition, practicality of most instruments is poorly described.

Among the volume instruments, validity is properly investigated but reliability is not. Only for filling material 'saline' reliability was investigated, and intra-rater reliability was good but inter-rater reliability was not good. The four general problems with filling instruments are definition of wound boundaries, wound flexibility due to slight movements of the patient, natural curvatures of the human body and cavity formation due to fibrotic scars of a healed wound. ${ }^{26}$ Moreover, filling material instruments are only useful for grades III and IV pressure ulcers and not for grades I and II. The time needed to apply filling material instruments is poorly investigated but is probably prohibitive for a bedside test. The ruler instrument is validated, but reliability and practicality are not properly investigated. This instrument is useful for grades I-IV ulcers and the time needed is not investigated but seems reasonable.

The surface instruments have been reasonably well validated. They are, however, only useful for grades I-II and not for grades III-IV ulcers. Wound tracings with a planimeter is highly accurate but requires substantial expertise and equipment. ${ }^{3,13}$ The time needed to use these instruments is not investigated but seems reasonable. Tracings are probably more time consuming than ruler length and width.

The scales 'PSST', 'PUSH' and the 'Sessing scale' give a description of the ulcer combined with a length/width/ undermining (PSST) and length/width (PUSH) measurement. The test time for ' $\mathrm{PUSH}$ ' and the Sessing scale is relatively short, but the time needed for the calculation of the score is prohibitively long for a bedside instrument. The PSST provides the most complete description of the ulcer according to the CPG recommendations.

\section{Conclusion}

Many pressure ulcer measurement instruments are not feasible in routine bedside practice due to the time needed for measurement and registration. For bedside measurement of the healing process in pressure ulcers in SCI patients, we advocate the use of the ruler method. To describe this process, we advocate the Sessing scale. Their clinimetric properties (validity and reliability) are promising, but further evaluation of these instruments is necessary.

\section{Conflict of interest}

The authors declare no conflict of interest. 


\section{References}

1 McKinley WO, Jackson AB, Cardenas DD, De Vivo MJ. Long-term medical complications after traumatic spinal cord injury: a regional model systems analysis. Arch Phys Med Rehabil 1999; 80: 1402-1410.

2 Raghavan P, Raza WA, Ahmed YS, Chamberlain MA. Prevalence of pressure sores in community sample of spinal injury patients. Clin Rehabil 2003; 17: 879-884.

3 Ferrell BA. Assessment of healing. Clin Geriatr Med 1997; 13: 575-586.

4 Consortium for Spinal Cord Medicine. Pressure Ulcer Prevention and Treatment Following Spinal Cord Injury. A Clinical Practice Guideline for Health Care Professionals. Paralyzed Veterans of America: Washington DC, 2001.

5 Emparanza JI, Aranegui P, Ruiz MM, Perez E. A simple severity index for pressure ulcers. J Wound Care 2000; 9: 86-90.

6 Pedley GE. Comparison of pressure ulcer grading scales: a study of clinical utility and inter-rater reliability. Int J Nurs Stud 2004; 41: 129-140.

7 Thomas DR. Existing tools: are they meeting the challenges of pressure ulcer healing? Adv Wound Care 1997; 10: 86-90.

8 Woodbury MG, Houghton PE, Campbell KE, Keast DH. Pressure ulcer assessment instruments: a critical appraisal. Ostomy Wound Manage 1999; 45: 42-55.

9 Harding KG. Methods for assessing change in ulcer status. Adv Wound Care 1995; 8: 37s-42s.

10 Mullins M, Thomason SS, Legro M. Monitoring pressure ulcer healing in persons with disabilities. Rehabil Nurs 2005; 30: 92-99.

11 Schubert V, Zander M. Analysis of the measurement of four wound variables in elderly patients with pressure ulcers. $A d v$ Wound Care 1996; 9: 29-36.

12 Berg W, Traneroth C, Gunnarsson A, Lossing C. A method for measuring pressure sores. Lancet 1990; 335: 1445-1446.

13 Cutler NR, George R, Seifert RD, Brunelle R, Sramek JJ, McNeill K et al. Comparison of quantitative methodologies to define chronic pressure ulcer measurements. Decubitus 1993; 6: 22-30.

14 Resch CS, Kerner E, Robson MC, Heggers JP, Scherer M, Boertman JA et al. Pressure sore volume measurement, a technique to document and record wound healing. J Am Geriatr Soc 1988; 36: $444-446$.

15 Hayward PG, Hillman GR, Quast MJ, Robson MC. Surface area measurement of pressure sores using wound molds and computerized imaging. J Am Geriatr Soc 1993; 41: 238-240.

16 Kundin JI. A new way to size up a wound. Am J Nurs 1989; 89: 206-207.

17 Bohannon RW, Pfaller BA. Documentation of wound surface area from tracings of wound perimeters. Phys Ther 1983; 63: $1622-1624$.

18 Lucas C, Classen J, Harrison D, De Haan RJ. Pressure ulcer surface area measurement using instant full-scale photography and transparency tracings. Adv Skin Wound Care 2002; 15: 17-23.

19 Griffin JW, Tolley EA, Tooms RE, Reyes RA, Clifft JK. A comparison of photographic and transparency-based methods for measuring wound surface area. Phys Ther 1993; 73: 117-122.

20 Thomas AC, Wysocki AB. The healing wound: a comparison of three clinically useful methods of measurement. Decubitus 1990; 3: $18-25$.

21 Palmer RM, Ring EFJ, Ledgard L. A digital video technique for radiographs and monitoring ulcers. J Photogr Sci 1989; 37: 65-67.

22 Russell L, Reynolds T, Carr J, Evans A, Holmes M. A comparison of healing rates on two pressure-relieving systems. Br J Nurs 2000; 9: 2270-2280.
23 Bates-Jensen BM, Vredevoe DL, Brecht M. Validity and reliability of the Pressure Sore Status Tool. Decubitus 1992; 5: 20-28.

24 Stotts NA, Rodeheaver GT, Thomas DR, Frantz RA, Bartolucci AA, Sussman $\mathrm{C}$ et al. An instrument to measure healing in pressure ulcers: development and validation of the Pressure Ulcer Scale for Healing (PUSH). J Gerontol A Biol Sci Med Sci 2001; 56: M795-M799.

25 Ferrell BA. The Sessing scale for measurement of pressure ulcer healing. Adv Wound Care 1997; 10: 78-80.

26 Plassmann P, Melhuish JM, Harding KG. Methods of measuring wound size: a comparative study. Ostotomy Wound Manage 1994; 40: $50-60$

27 Covington JS, Griffin JW, Mendius RK, Tooms RE, Clifft JK. Measurement of pressure ulcer volume using dental impression materials: suggestion from the field. Phys Ther 1989; 69: 690-694.

28 Hooker EZ, Sibley P, Nemchausky B, Lopez E. A method for quantifying the area of closed pressure sores by sinography and digitometry. J Neurosci Nurs 1988; 20: 118-127.

29 Bates-Jensen BM. The Pressure Sore Status Tool, a few thousand assessments later. Adv Wound Care 1997; 10: 65-73.

30 Bates-Jensen B. The Wound Intelligence System: early issues and findings from multi-site tests. Ostomy Wound Manage 1996; 42 (10Asuppl): 53s-61s.

31 Bates-Jensen BM. Toward an intelligent wound assessment system. Ostomy Wound Manage 1995; 41 (7A): 80s-87s.

32 Maklebust J. PUSH Tool reality check: audience response. $A d v$ Wound Care 1997; 10: 102-106.

33 Bartolucci AA, Thomas DR. Using principal component analysis to describe wound status. Adv Wound Care 1997; 10: 93-95.

34 Pompeo M. Implementing the PUSH Tool in clinical practice: revisions and results. Ostomy Wound Manage 2003; 49: 32-46.

35 Gardner SE, Frantz RA, Bergquist S, Shin CD. A prospective study of the pressure ulcer scale for healing (PUSH). J Gerontol A Biol Sci Med Sci 2005; 60A: 93-97.

36 Berlowitz DR, Ratliff C, Cuddigan J, Rodeheaver GT. The PUSH tool: a survey to determine its perceived usefulness. Adv Skin Wound Care 2005; 18: 480-483.

37 Ferrell BA, Artinian BM, Sessing D. The Sessing scale for assessment of pressure ulcer healing. Am Geriatr Soc 1995; 43: $37-40$.

38 Ferrell BA, Keeler E, Siu AL, Ahn S, Osterweil D. Cost-effectiveness of low-air-loss beds for treatment of pressure ulcers. J Gerontol A Biol Sci Med Sci 1995; 50A: M141-M146.

39 Robson MC, Maggi SP, Smith PD, Wassermann RJ, Mosiello GC, Hill DP et al. Ease of wound closure as an endpoint of treatment efficacy. Wound Rep Reg 1999; 7: 90-96.

40 Houghton PE, Kincaid CB, Campbell KE, Woodbury MG, Keast DH. Photographic assessment of the appearance of chronic pressure and leg ulcers. Ostomy Wound Manage 2000; 46: 20-30.

41 Sussman C, Swanson G. Utility of the Sussman Wound Healing Tool in predicting wound healing outcomes in physical therapy. Adv Wound Care 1997; 10: 74-77.

42 Sanada H, Moriguchi T, Miyachi Y, Ohura T, Nakajo T, Tokunaga $\mathrm{K}$ et al. Reliability and validity of DESIGN, a tool that classifies pressure ulcer severity and monitors healing. J Wound Care 2004; 13: $13-18$

43 Frantz RA, Johnson DA. Stereophotography and computerized image analysis: a three-dimensional method of measuring wound healing. Wounds 1992; 4: 58-64.

44 Sussman C. Presenting a draft pressure ulcer scale to monitor healing. Adv Wound Care 1997; 10: 92. 